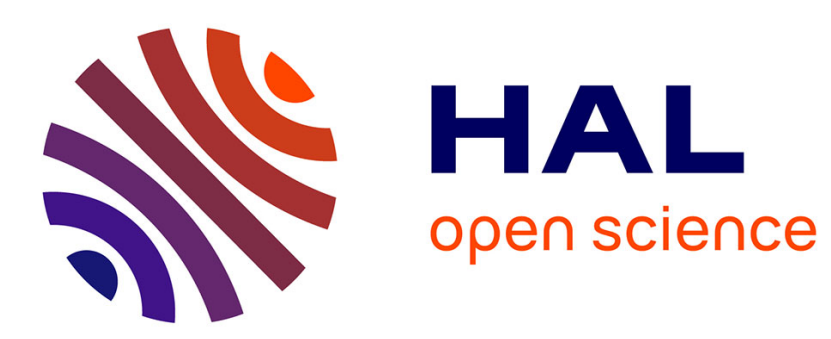

\title{
La ruée vers l'or jaune : difficultés de l'engagement scientifique dans un mouvement social
}

Quentin Ravelli

\section{To cite this version:}

Quentin Ravelli. La ruée vers l'or jaune : difficultés de l'engagement scientifique dans un mouvement social. Sociologie, 2020, 11 (3), pp.267. 10.3917/socio.113.0267 . hal-03101134

\section{HAL Id: hal-03101134 \\ https://hal.science/hal-03101134}

Submitted on 7 Jan 2021

HAL is a multi-disciplinary open access archive for the deposit and dissemination of scientific research documents, whether they are published or not. The documents may come from teaching and research institutions in France or abroad, or from public or private research centers.
L'archive ouverte pluridisciplinaire HAL, est destinée au dépôt et à la diffusion de documents scientifiques de niveau recherche, publiés ou non, émanant des établissements d'enseignement et de recherche français ou étrangers, des laboratoires publics ou privés. 


\section{La ruée vers l'or jaune : \\ difficultés de l'engagement scientifique dans un mouvement social}

The yellow gold rush: difficulties of scientific engagement in a social movement

Sociologie, 2020, $\mathrm{n}^{\circ} 3$, vol. 11, 267-275

Surgi au mois de novembre 2018 pour contester une taxe sur le carburant imposée par le gouvernement, le mouvement des Gilets jaunes s'est transformé en soulèvement de masse contre les taxes, les injustices sociales et les inégalités économiques. Il n'a pas seulement bouleversé ceux qui se trouvaient sur la ligne de front, qu'il s'agisse des manifestants, des occupants de ronds-points et des militants, ou encore, de l'autre côté de la barricade, des notables, des hommes politiques et des forces de l'ordre. Il a aussi transformé, à des degrés divers, une série de professions, comme les avocats, les responsables syndicaux, les journalistes ou les chercheurs, simultanément happés et remis en cause par ce soulèvement. Certains journalistes ont été expulsés des rassemblements, comme ceux de BFM TV, tandis que d'autres les défendaient avec détermination, comme Taranis News. Les cadres syndicaux des échelons intermédiaires - comme les secrétaires d'unions locales - ont été à la fois fustigés et intégrés aux manifestations. Le monde des chercheurs, en particulier en sciences sociales, ne fait pas exception : en tant qu'experts proches des médias et du pouvoir, ils ont été pris à partie et attaqués ; en tant que sympathisants ou compagnons de route des Gilets jaunes, ils ont pu se fondre dans la mobilisation et la construire activement. Dans un tel contexte d'effervescence, les chercheurs ont oscillé - et oscillent encore - entre une science embarquée et une science engagée dans la lutte, avec un large éventail de situations intermédiaires. La première est emportée par un courant face auquel elle s'efforce de maintenir sa neutralité sans y parvenir, s'exposant ainsi aux influences politiques et médiatiques, aux Gilets jaunes ou à leurs critiques, au risque de ne plus être objective. La seconde cherche à s'enraciner dans le mouvement, à y prendre parti pour y construire une objectivité située.

La force d'attraction des Gilets jaunes n'a pas seulement infléchi les parcours individuels, même si certains se sont retrouvés dans des situations inédites, loin de leurs pratiques habituelles, emportés dans ce nouvel élan qu'ils cherchaient à décrypter, souvent sans y arriver. Collectivement, les frontières disciplinaires, les choix théoriques et méthodologiques se sont, eux aussi, déplacés, en se confrontant au fil des rencontres, des collaborations et des prises de position, avec leurs inévitables lots de violence symbolique. Les tendances de l'approche par observation - directe ou indirecte, participante ou détachée, ouverte ou à couvert, avec ou sans gilet jaune - incarnent bien comment un puissant mouvement social bouleverse le monde de la recherche, en opérant des rapprochements et des 
clivages imprévus. Les statistiques, denrée rare et précieuse - même quand il s'agit moins de la politique des « grands nombres » analysée par Alain Desrosières (2014 [1993]) que de « petits nombres » patiemment collectés - ont nourri cette genèse accélérée d'un champ soumis, dès la naissance, à des injonctions contradictoires : publier vite pour ne pas être dépassé et avoir sa part de soleil, mais en prenant le temps de comprendre une lutte inhabituelle ; travailler collectivement pour avoir une vision générale du mouvement, mais en veillant à construire sa carrière individuelle en préservant ses données ; être objectif, mais sans jamais pouvoir être neutre, au risque de confondre l'un avec l'autre. À partir d'une description de cette ruée vers l'or jaune, avec ses grandeurs et ses petitesses, on a essayé de tracer une voie possible, en s'engageant d'abord, au long cours et en portant un Gilet jaune, pour chercher ensuite à comprendre le mouvement, à penser sa propre position dans un mouvement d'ensemble. Après un panorama des méthodes adoptées, et avant de présenter quelques caractéristiques générales du champ scientifique des Gilets jaunes, avec ses violences et ses contradictions, je présenterai le chemin que j'ai suivi, engagé dans le mouvement et pour le mouvement avec un Gilet jaune - c'est l'une des voies possibles, parmi tant d'autres.

\section{Science engagée et science embarquée : un éventail de méthodes}

Le mouvement des Gilets jaunes n'a, bien sûr, pas fait naître de nouvelles formes de recherche. Mais en attirant à lui et en déviant de leur cours de nombreux chercheurs - poussés par leur curiosité, leur carrière, leurs convictions ou les trois à la fois - il a constitué un creuset inédit, où des façons de comprendre la société qui avaient tendance à coexister de façon pacifique, en s'ignorant, ont été immergées dans un même bain. Elles y ont fabriqué des variétés hybrides. Une des manières d'identifier les positions dans ce nouveau milieu consiste à s'intéresser au moment où le chercheur entre dans le monde du mouvement. Ce monde, façonné par la télévision, la presse et les débats politiques, est distinct, et souvent extérieur, à la vie interne du mouvement. C'est pourtant dans cette sphère que sont nées la plupart des analyses - ce qui ne veut pas forcément dire qu'elles sont fausses ni que, inversement, le fait de participer au mouvement lui-même offre une quelconque garantie d'objectivité.

Même si ce monde du mouvement est structuré par des discours médiatiques, par l'influence d'un appareil d'État hostile et de partis qui cherchent à discréditer ou à récupérer la contestation, le fait d'avoir mené des recherches antérieures sur des sujets proches permet d'y voir plus clair et de ne pas se laisser influencer. En ce qui me concerne, les travaux sur les crises économiques et les mouvements sociaux, en particulier en Espagne où les classes populaires, déjà révoltées en dehors des cadres syndicaux habituels, se sont mobilisées contre les banques (Ravelli, 2017, 2019), ainsi qu'une connaissance des formes de contestation internes aux grandes entreprises et de leurs limites (Ravelli, 2015), m'ont servi de boussole. Les textes de Gérard Noiriel et Nicolas Truong (2018), depuis une perspective d'historien des classes populaires, d'Alexis Spire $(2018,2019)$, à partir d'une sociologie historique des résistances à l'impôt, ou de Laurent Jeanpierre (2019), après s'être intéressé aux révoltes des pays arabes, font partie de cette façon d'aborder le mouvement. Foisonnante, on y croise de 
nombreuses disciplines, de l'histoire - Sophie Wahnich, Samuel Hayat - à la philosophie Étienne Balibar -, en passant par l'urbanisme - Aurélien Delpirou - et l'économie - Thomas Piketty (Confavreux, 2019) : la liste est longue car c'est une grande partie du champ intellectuel français qui s'est prêté à l'exercice, avec des résultats contrastés, de la comparaison minutieuse au hâtif billet d'humeur recyclant quelques coupures de presse.

\section{Dès l'origine : une bataille de chiffres}

Très vite - en réalité dès le 17 novembre mais surtout au début du mois de décembre 2018 - le besoin de comprendre la situation est devenu une urgence politique d'autant plus pressante que le mouvement réussissait à faire trembler l'appareil d'État. Cette situation induisait une demande croissante pour des informations étayées empiriquement, aussi bien du côté des opposants que des contestataires, plongés dans les incertitudes. Cette attente médiatique était attisée par la multiplicité des interprétations, souvent contradictoires, que les chercheurs étaient censés valider ou infirmer : s'agissait-il d'un mouvement d'extrême droite ou d'une variété méconnue du mouvement ouvrier, d'une violence majoritaire ou minoritaire, offensive ou défensive, de classes populaires ou d'une petite bourgeoisie déclassée, de groupes politisés ou sans passé militant? L'interprétation générale du mouvement, et des attitudes politiques à adopter, était d'autant plus difficile à cerner que les sources syndicales habituelles faisaient défaut : seuls les chiffres du ministère de l'Intérieur existaient pour les manifestations, jusqu'à ce qu'apparaisse la comptabilité indépendante du Nombre jaune, souvent dépassée par celle de Policiers en colère. ${ }^{1}$

Cherchant à peser dans le débat, des questionnaires ont commencé à alimenter l'arène médiatique et à solidifier des interprétations politiques divergentes. À une enquête dite « pionnière », présentant les Gilets jaunes comme une " révolte des revenus modestes » orientée à gauche, publiée dans Le Monde dès le 11 décembre 2018, ${ }^{2}$ à partir de 166 questionnaires largement étoffés ensuite (Collectif d'enquête sur les Gilets jaunes 2018 puis 2019), répondent des publications fondées sur un « échantillon représentatif de 2116 Français $\cdot e \cdot s$ » du Cevipof de Sciences Po, ${ }^{3}$ dont certaines concluent, par exemple dans The Conversation« l'expertise universitaire, l'exigence journalistique »- au poids majoritaire du vote pour le Rassemblement National, voire à une « transition populiste de droite », ${ }^{4}$ à partir d'un panel qui ne porte pourtant pas sur les Gilets jaunes eux-mêmes mais sur leurs « soutiens ». D'autres études statistiques, critiquant ce biais méthodologique, arrivent ensuite en renfort

\footnotetext{
${ }^{1}$ Pour l'Acte XX des Gilets jaunes, par exemple, les estimations étaient respectivement de 33 700, 103713 et 120000 manifestants.

${ }^{2}$ Le Monde, " "Gilets jaunes" : une enquête pionnière sur la "révolte des revenus modestes" ", 11 décembre 2018, https://www.lemonde.fr/idees/article/2018/12/11/gilets-jaunes-une-enquete-pionniere-sur-la-revolte-desrevenus-modestes_5395562_3232.html.

${ }^{3}$ Yann Algan et al., «Qui sont les Gilets jaunes et leurs soutiens ? », Note de l'Observatoire du Bien-être, no 2019-03, https://www.cepremap.fr/2019/ 02/note-de-lobservatoire-du-bien-etre-n2019-03-qui-sont-les-giletsjaunes-et-leurs-soutiens/.

${ }^{4}$ Luc Rouban, "Les "gilets jaunes", une transition populiste de droite », The Conversation, 28 janvier 2019, https://theconversation.com/les-giletsjaunes- une-transition-populiste-de-droite-110612.
} 
pour faire pencher la balance dans l'autre sens, comme celle du collectif Quantité critique (2018), dans L'Humanité du 19 décembre puis dans Médiapart, le 19 avril 2019, à partir de 526 questionnaires passés en ligne entre le 23 et le 30 novembre. $^{5}$

Dans cette bataille de chiffres pour l'interprétation hégémonique du mouvement, on notera la part belle aux études à distance ${ }^{6}$ ou à partir des groupes Facebook (Boyer et al., 2019, sur les « déterminants de la mobilisation »). Le risque de cette méthode, quand elle est utilisée seule et indépendamment d'une connaissance fine des luttes sociales, est de faire surgir des données déformées par les réseaux sociaux : par exemple, que signifie l'idée qu'il y aurait eu « 788 points de blocages » au 17 novembre 2018 (Boyer et al., 2019, p. 1, 7), si ce chiffre est celui qui est officiellement déclaré la veille sur le site blocage17novembre.fr, où n'apparaît pas l'écrasante majorité des ronds-points mobilisés, et que le ministère de l'intérieur lui-même dénombrera 2000 rassemblements, un chiffre certainement sous-évalué ? Plus généralement, que penser de l'échelle de classement des opinions politiques de 1 à 7 , de l'extrême droite à l'extrême gauche, quand les répondants refusent le principe même de cette séparation?

\section{L'arbre ethnographique des Gilets jaunes}

C'est sur un registre bien distinct - qui dans l'idéal devrait être complémentaire du premier mais l'est rarement - que se sont déployées des approches par observation, dont le spectre n'est pas moins étendu que celui des statistiques. Elles ont cependant un point commun que n'ont pas entre elles les démarches quantitatives : comme en médecine, l'accumulation de chiffres ne saurait remplacer le regard anatomique, essentiel à la naissance de la clinique dont le regard s'engage dans le corps, selon Michel Foucault (2015 [1963]). De même, pour comprendre un mouvement, encore faut-il le voir de l'intérieur, sur la durée, pour connaître ses racines et raisons profondes dans un petit groupe d'interconnaissances et éviter ainsi l'extrapolation à partir d'échantillons insuffisants ou non représentatifs, dont l'un des risques est de refléter la cartographie du monde académique - plus présent dans les grandes villes, plus à l'aise chez les militants, désarçonné par les prolétaires révoltés - plutôt que celle des Gilets jaunes. Croyant voir son objet, le chercheur se voit en fait lui-même, dans un miroir de chiffres dont il n'a pas conscience, sur lequel il projette non seulement son désir de savoir, sa libido sciendi, mais aussi ses attentes politiques et professionnelles.

Cependant, l'objectivité des résultats n'est pas plus aléatoire pour les chiffres que pour les observations. Tout dépend de l'angle, de la durée, de la continuité, des choix d'implantations, de la minutie de la retranscription et, pourrait-on dire, de l'opiniâtreté contre

\footnotetext{
${ }^{5}$ Collectif Quantité critique, « Enquête. Les gilets jaunes ont-ils une couleur politique ? », L’Humanité, 19 décembre 2018, https://www.humanite.fr/ enquete-les-gilets-jaunes-ont-ils-une-couleur-politique-665360 ; idem, « Gilets jaunes : à qui va profiter le mouvement ? », 19 avril 2019, https:// www.mediapart.fr/journal/france/190419/gilets-jaunes-qui-va-profiter-lemouvement.

${ }^{6}$ Voir par exemple, sur un panel de 100 répondants au niveau de dix villes différentes, Simon Porcher, « "Gilets jaunes" : une fracture nord-sud ? », The Conversation, 14 novembre 2019, https://theconversation.com/giletsjaunesune- fracture-nord-sud-126962.
} 
soi-même, au sens d'une volonté d'aller à l'encontre de ses propres projections - on retrouve ici l'usage « kamikaze » de l'hypothèse, détaillée par Michael Burawoy (2009) ${ }^{7}$. Dans l'arbre des méthodes ethnographiques, on rencontre d'abord une première bifurcation : l'observation directe ou indirecte, la première s'appuyant sur une expérience de terrain en chair et en os, la seconde reposant sur des sources secondaires, comme les innombrables photos, films, lives, documentaires et reportages, par exemple les 8500 photos des inscriptions aux dos des Gilets jaunes, dont 365 ont été retenues dans un livre (Collectif Plein le dos, 2020). Parmi les nombreux exemples d'observation directe, on peut noter les approches de Benoît Coquard (2018) et de Raphaël Challier (2019), qui ont en commun d'avoir vu surgir le mouvement des Gilets jaunes dans des régions rurales de l'Est de la France, où ils ont fait leurs thèses de sociologie sur « ceux qui restent » malgré l'exode rural (Coquard, 2019) et les " simples militants » du FN, des JC et de l'UMP en milieu populaire (Challier, 2018). Ils s'apparentent donc plutôt aux « pêcheurs », qui étaient déjà sur place quand l'événement a surgi, qu'aux « chasseurs », qui lui courent après, pour reprendre la distinction employée chez les réalisateurs de documentaires. Souvent, la recherche se réoriente en cours de route, pendant la thèse, comme c'est le cas de Serena Boncompagni pour l'anthropologie politique de la région d'Hénin-Beaumont ou Charif El Alaoui sur les mouvements sociaux au Maghreb - tous deux aspirés par les Gilets jaunes. Ensuite, parmi les observations directes, se distinguent l'observation directe non participante et l'observation directe participante, ${ }^{8}$ la première permettant par exemple d'aller en manifestation « pour voir », discuter et prendre des notes, mais sans Gilet jaune, tandis que la seconde suppose d'être avec les Gilets jaunes. Comme le disent Bernard Floris et Luc Gwiazdzinski (2019, p. 195) : « nous n’aurions jamais imaginé, en démarrant ce projet, [...] qu'il nous conduirait à préparer une soupe aux lentilles, à repérer des coins à muguet, à participer à un "safari-pêche", à planter des tournesols ou à danser et chanter sur l'air de "Tié la famille" ». Au-delà de ce tableau bucolique, être « avec » les Gilets jaunes, c'est essuyer des tirs de grenade, se lever à l'aube pour bloquer des raffineries, empêcher des barrages d'être forcés par des poids lourds, contacter des avocats, risquer de la prison ferme - tout dépend de quoi le « avec » signifie véritablement.

De fait, dans la participation à un mouvement, il y a là encore des degrés qui deviennent, ici, des différences de nature, car une démarche retenue, d'accompagnement, se distingue d'une autre, engagée, militante. La première est la plus courante et impose une barrière entre les observés et l'observateur, qui est présent pour d'autres raisons que la colère des Gilets jaunes. La seconde cherche une voie hybride qui peut se revendiquer de la recherche-action, comme c'est le cas de « l'Atelier des ronds-points » coordonné par Benoît Hazard : « Dans une démarche de recherche-action assumant que les acteurs du mouvement sont des producteurs de savoirs, nous essayerons d'avancer sur les modalités de mise en forme de ces récits » (L’Atelier des Ronds-Points, 2019). Invitant des Gilets jaunes à l'Ehess,

\footnotetext{
${ }^{7}$ Cet usage suppose d'être capable de voir, même quand on est persuadé d'avoir affaire à des groupes d'extrême droite, que la plupart des manifestants n'ont aucune expérience militante préalable et refusent les clivages entre la gauche et la droite, ou encore de reconnaître qu'il y a une multitude de petits groupes politiques actifs, même quand on est soi-même convaincu qu'il s'agit d'une révolte de milieux populaires non politisés.

${ }^{8}$ Participantes ou non, ces observations peuvent se faire à découvert, quand on se présente comme chercheur, ou à couvert, ce qui se pratique parfois dans des milieux hostiles mais pose de nombreuses difficultés éthiques.
} 
l'atelier reprend à son compte une partie du manifeste du Laboratoire d'innovation sociale par la recherche-action, qui cherche à « modifier l'orientation historique » des « rapports de production et reproduction » par la « constitution de minorités actives » et cherche à ouvrir des espaces d'expression politique à l'intérieur des institutions (LISRA, sd). Il inverse le sens habituel de l'engagement du chercheur, qui laisse alors l'engagement se produire chez lui, dans l'ambitieuse idée d'une « co-production » mi-savante, mi-engagée, semée de malentendus croisés à désamorcer.

Si les ethnographies, quelle que soit leur place dans cet arbre méthodologique, sont le plus souvent conduites de façon artisanale et isolée, le mouvement des Gilets jaunes les a forcées à s'inscrire dans un cadre collectif, en imposant ce qu'on peut appeler une ethnographie multi-située rétrospective, car elle se fabrique une fois passé l'engagement sur le terrain, par comparaison. Chacun perçoit en effet les limites d'une recherche sur un seul lieu de manifestation, qu'il s'agisse du Puy-en-Velay comme des Champs-Élysées, ou sur un seul rond-point dont la sociologie dépend, entre autres, de la proximité et de la taille des villes, ou de leur éloignement, des taux de chômage, des micro-climats politiques, des directives préfectorales. Il faut toujours « élargir » l'étude de cas élargie, comme le suggère l'école de Manchester, selon Andreas Glaeser (2006). Même une simple collection de recherches sur des ronds-points séparés, juxtaposant des contextes hétérogènes, reste incapable d'opérer le saut nécessaire de la synthèse, ni d'en percevoir le degré de généralité. Il y a donc des efforts, encore en cours, pour comparer des travaux qualitatifs, ce qui est, on le conçoit, encore plus compliqué que de comparer des chiffres. Comment s'orienter dans cet écheveau de chemins possibles?

\section{Un chemin en Gilet jaune : de l'Est du Loiret aux Adas, via les Champs-Élysées}

Le mouvement des Gilets jaunes s'est structuré autour de deux grandes lignes : la base arrière essentielle des ronds-points, instaurant des rapports de force locaux ; les grandes offensives des manifestations du samedi, dont l'épopée a rythmé le temps médiatique pendant près d'un an, dans l'attente d'une faille dans l'appareil d'État ou d'un essoufflement de la protestation. Comprendre précisément un tel mouvement en négligeant l'un des deux moments de ce moteur à deux temps aurait été arbitraire et restrictif, car je souhaitais justement cerner comment les classes populaires, par un ralliement inhabituel et fondamentalement autonome, pouvaient ainsi se projeter, apparemment sans expérience préalable, contre le pouvoir d'État avec une telle détermination. Le choix que j'ai fait a donc consisté à ne pas choisir, mais à faire comme de nombreux Gilets jaunes : rond-point en semaine, manifestation le week-end. Ce choix a dérivé de l'engagement dans le mouvement, où je me suis considéré d'abord comme Gilet jaune et ensuite comme chercheur, ce qui permettait de comprendre avec plus de nuance un mouvement dans lequel les convictions politiques, les choix personnels et les rapports de confiance se développent prudemment, en s'effeuillant, sans s'ouvrir au premier venu. Sans surprise, la meilleure manière de comprendre qui sont les Gilets jaunes est de commencer par en porter un - mais où s'installer ? L'avantage de l'Est du Loiret, que je connaissais déjà, est que Paris n'est pas loin et qu'on 
peut donc voir à l'œuvre, d'une scène à l'autre, la courroie de transmission qui propulse la contestation des campagnes populaires aux pieds du pouvoir présidentiel. Ce ne sont que 128 $\mathrm{km}$ qui séparent la place de l'Étoile du rondpoint d'Amilly, devenu, depuis le 17 novembre 2018, l'un des centres de la mobilisation de l'Est du Loiret pendant plusieurs mois.

Ces deux espaces ne sont pourtant pas équivalents. Certes, la «montée sur Paris » est un moment puissant, qui participe de la confrontation centrale avec l'État, agrège des milieux militants divers dont les rencontres modifient la composition et l'orientation du mouvement. Les manifestations du samedi façonnent ainsi des expériences politiques nouvelles, où les ouvriers et artisans des régions rurales peuvent par exemple se rapprocher des black blocs formés dans les cortèges de tête contre la loi Travail, et inversement. De ce point de vue, il y avait un sens à être présent à Paris le samedi, le plus souvent possible, aux « actes » où se réactivait la longue histoire des révoltes parisiennes, comme au jardin des Tuileries lors de l'acte IV du samedi 8 décembre 2018, où l'espace de la mobilisation s'est étendu dans plusieurs quartiers au Nord de la place Concorde. Il fallait aussi suivre les coulisses des actes : la répression policière et judiciaire, en particulier lors des comparutions immédiates et des procès suivant les rassemblements. Mais comme je me rendais dans le Loiret, en parallèle, il m'est rapidement apparu que même les rassemblements centraux s'expliquaient par l'arrivée de nouveaux manifestants qui ne connaissaient pas les us et coutumes des manifestations parisiennes : l'absence souvent soulignée de cortèges, de services d'ordres et de banderoles syndicales avait des racines sociologiques introuvables dans la capitale. J'essayais donc, dès que possible, d'aller aux manifestations des Champs-Élysées avec les Gilets jaunes du Loiret : même à Paris, les ronds-points restaient la matrice du mouvement, le lieu où se fabriquait et se maintenait une colère nourrie des faits d'armes hebdomadaires.

En périphérie d'une petite ville du centre de la France, au taux de chômage élevé, où les industries ferment ou se restructurent en éliminant les contestations internes, où on assiste à la suppression progressive des services publics touchant de nombreux aspects de la vie sociale - des transports à la poste en passant par la santé - et où l'abstention, les votes RN et FI sont élevés, l'espace du soulèvement ressemble à de nombreuses autres régions de France où les Gilets jaunes se sont révoltés contre les inégalités sociales. Mais dès qu'on entre dans le détail des spécificités locales, les perspectives changent. On sort des généralités pour découvrir des formes d'organisation concrètes et inventives : la particularité de la portée non par un seul rond-point, ou plusieurs ronds-points isolés, mais par un maillage de ronds-points en interaction constante, formant une « grappe giratoire » (Ravelli, 2020) permettant la coexistence de différentes tendances idéologiques, qui désarçonne commentateurs et statisticiens.

Dès le départ, avant même d'aller pour la première fois sur un rond-point, dans les discussions préalables que j'ai eues à Montargis pour savoir où me rendre et qui rencontrer, ce n'est pas un rond-point mais deux - Châlette et Cacahuète - qui m'ont été décrits. Leurs identités, ou plutôt ce qu'on pourrait appeler leurs « personnalités sociales » respectives, s'avéraient étonnamment interchangeables : tantôt ceux de Châlette étaient raisonnables et ceux de Cacahuète radicaux, tantôt c'était l'inverse ; parfois le premier rond-point était « 
facho » et l'autre « gaucho », et parfois c'était l'opposé. Une fois sur place, la situation ne s'est pas éclairée car ces différentes propriétés semblaient en fait combinées sur chaque rondpoint, et non réparties entre eux. À force de rencontres, en me mettant ensuite à vivre sur place au bout de quelques mois, je me suis aperçu que les ronds-points se façonnaient par des séries d'interactions fluides - émulation, rivalité, coordination... - entre lesquels certains Gilets jaunes se déplaçaient, opérant des changements de personnalité sociale des rondspoints. La grappe giratoire de Montargis incluait deux autres ronds-points importants Chesnoy et Zébra - et la force du mouvement résidait en fait dans sa capacité à faire, de ces grappes, des structures politiques souples. Inédites, ces structures fonctionnent bien différemment des assemblées générales, qui incarnent souvent la cellule élémentaire des mouvements sociaux, comme c'est le cas par exemple chez les étudiants ou dans les grèves ouvrières. Localement, il y avait certes des réunions, à un rythme régulier, mais elles n'étaient pas le socle du mouvement et avaient tendance à aiguiser les tensions au lieu de les apaiser, en réveillant et formalisant les désaccords politiques.

Comment expliquer, alors, que de nombreux ronds-points aient pourtant cherché à se structurer dans des coordinations nationales appelées des « Assemblées des assemblées », tandis que d'autres, plus nombreux, comme ceux du Loiret, les ignoraient ? Cette énigme de la coordination et de la convergence, fondamentale pour la structuration d'un mouvement, pose la question de la généralisation des observations. Les statistiques permettent d'éclairer en partie cette question. J'ai donc fait passer, avec d'autres chercheurs et étudiants, des questionnaires, non seulement aux Gilets jaunes mais aux Assemblées des assemblées (Adas) 9. Il aurait été possible de construire de nouveaux questionnaires, mais cela aurait empêché des comparaisons ultérieures avec des données déjà patiemment collectées. Il semblait donc préférable de s'inscrire dans une démarche de recherche cumulative, en utilisant les questions de questionnaires déjà existants, pour les distribuer, malgré leurs défauts, aux Gilets jaunes des assemblées, afin d'enrichir une base de données déjà existante - celle du Collectif d'enquête sur les Gilets jaunes mentionné plus haut - pour que les données puissent être plus tard comparées et contrastées avec celles des ronds-points et des manifestations 10. Les 438 questionnaires distribués aux Adas permettent de mieux comprendre les formes de coordination du mouvement, la distance qui sépare certaines sphères militantes des classes populaires mais surtout le véritable effort de coordination et d'ancrage dans le mouvement de ces Adas. Intégrées aux travaux de recherche statistique présentés plus haut, ces données contribuent à mieux cerner cette lutte sociale en la sortant de la caricature, en montrant qu'elle s'est déployée sur différents plans, partiellement interséquents, qui sont loin d'être une accumulation chaotique.

Malgré les tensions qui pèsent sur tout effort de recherche collective à vif, et qui sont liées à la valeur symbolique des données recueillies pour la construction des carrières dans le monde de la recherche, on peut espérer que ces données, une fois combinées, éclaireront l'ensemble de l'édifice construit par les Gilets jaunes. La complexité d'une telle démarche s'explique par la rapidité avec laquelle a surgi le champ de recherche sur les Gilets jaunes, qui est en partie un sous-produit de la mobilisation elle-même : tout en opérant des décloisonnements prometteurs entre les méthodes, les disciplines et d'autres champs extra- 
académiques, l'engagement scientifique se heurte aussi à des injonctions contradictoires et à des impasses théoriques.

\title{
Frontières disciplinaires et doubles contraintes : le surgissement accéléré d'un petit champ scientifique
}

Quel que soit le parcours que l'on trace dans cette mobilisation foisonnante, on y est forcément confronté à une double violence : celle du mouvement lui-même, heurté par une répression féroce, tant de fois soulignée, et celle de ceux qui le commentent, dont on parle moins souvent. Ces quelques notes, prises à l'entrée du tribunal le jour du procès d'Éric Drouet, en témoignent :

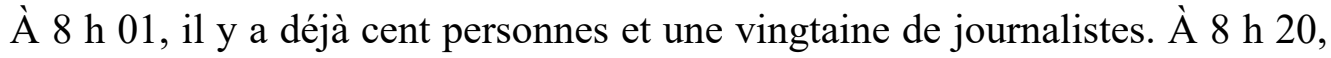
un gilet jaune apparaît au loin, et disparaît aussitôt. Il y a par contre plus de trente journalistes qui se ruent sur Éric Drouet et son avocat, avec une telle violence qu'une journaliste tombe par terre. Une vieille dame grommelle, « c'est pas Johnny, mais c'est pire que Johnny ! ». Pour voir au-dessus de ses confrères, un photographe déplie un petit tabouret téléscopique, et un homme qui comparait Macron à un dictateur quelques minutes auparavant commente, en regardant cet étrange meuble médiatique : « ça fait trente-cinq ans que je viens au tribunal, je n'ai jamais vu ça » (vendredi 15 février 2019).
\end{abstract}

Cette pression médiatique ne s'exerce pas seulement sur les journalistes et les Gilets jaunes, mais sur tous les métiers associés à la chaîne de fabrication de l'interprétation du mouvement, en particulier les chercheurs. En quelques semaines, s'est formé un petit champ scientifique. Au sens le plus classique du terme, ce champ repose sur l'organisation d'un marché de biens symboliques - observations, statistiques, analyses - dont la valeur bondit subitement. Cette hausse des cours permet d'accéder aux grands médias et d'atteindre deux objectifs liés entre eux : faire connaître ses idées plus largement et contribuer ainsi à une recherche engagée dans le débat public ; construire sa carrière en se faisant connaître et reconnaître, parfois de façon inespérée. Combinée à la force d'attraction du mouvement luimême, cette double opportunité déplace les frontières internes et externes des disciplines comme du champ lui-même, et fait naître des injonctions contradictoires.

Chez Pierre Bourdieu, les passages les plus éclairants pour comprendre la structure des champs sont ceux qui décrivent leur genèse, car celle-ci fait clairement apparaître, par une suite de crises de croissances, les rapports de domination structurale qui les sous-tendent, ainsi que les doubles contraintes qui pèsent sur eux. La «phase critique de l'émergence du champ », dans le cas de la littérature française du milieu du XIXe siècle, montre à la fois que les écrivains, comme Flaubert ou Baudelaire, revendiquent et construisent l'autonomie artistique - largement mythologique - du champ littéraire vis-à-vis des institutions bourgeoises, mais aussi que ce champ est structuré par ces mêmes institutions : les salons, qui exercent un pouvoir politique sur l'écrivain ; la presse, qui le soumet au pouvoir médiatique (Bourdieu, 1998 [1992], p. 85-118). 
Au gré des événements extérieurs dont elles se nourrissent, les sciences sociales voient naître des espaces scientifiques qui fonctionnent transitoirement comme des champs - le cas des Gilets jaunes, aussi passager qu'il se révélera peut-être, en est un bon exemple. Ses frontières externes, d'abord, ont modifié le rôle social habituel du chercheur. Les lignes entre les milieux académique et médiatique se sont brouillées, comme en témoignent deux livres coordonnés par des journalistes, Joseph Confavreux (2019), de Mediapart, et Sylvain Bourmeau (2019), d'AOC, où de nombreux chercheurs ont publié leurs analyses à chaud, quoique prudemment, depuis leurs disciplines respectives. La multiplication de ces interventions publiques, et de nombreuses autres, en clivant le milieu académique entre proet anti-Gilets jaunes, a aussi modifié les relations habituelles entre le pouvoir politique, contesté, et les milieux scientifiques sortant de leur réserve et s'engageant, parfois pour la première fois comme de nombreux Gilets jaunes, au cœur des débats publics.

À l'intérieur du champ, les rapports entre disciplines ont eux aussi évolué, faisant éclater certaines barrières et permettant de collaborer plus souvent avec d'autres disciplines que la sienne : l'importance accordée à l'espace local par les géographes devenait une question centrale pour les sociologues ou les anthropologues; le poids de l'histoire des mouvements sociaux devenait indispensable aux analyses d'anthropologues, au-delà du cercle des historiens ; les facteurs économiques s'avéraient inévitables, à tous, pour cerner un mouvement structuré par les inégalités de revenus et les injustices fiscales ; la créativité artistique des révoltés poussait les littéraires à s'intéresser aux slogans, aux cabanes, aux peintures des gilets, à l'art brut du mouvement social. Ces convergences se sont traduites par une première injonction contradictoire, assez évidente, qui est celle de la collaboration contrariée, rendant nécessaire le travail à plusieurs, pour réussir à comprendre un mouvement impossible à appréhender seul, tout en risquant d'y perdre sa propre perspective, dans un univers saturé d'interprétations.

Mais d'autres effets moins évidents méritent être soulignés, au premier chef ce qu'on pourrait appeler la synchronisation des carrières : en se retrouvant, à des âges différents, dans les mêmes mobilisations, parfois physiquement au coude-à-coude, étudiants et statutaires ont vu leurs relations modifiées. Le professeur émérite replongeant dans l'empirie croisait l'étudiant de Master accumulant en quelques mois suffisamment de matière première pour fabriquer une ou deux thèses de doctorat - l'un et l'autre travaillant ensemble en militant, ou inversement. Ce sont souvent, d'ailleurs, les solidarités plus politiques que scientifiques qui faisaient les ciments solides. Un tel nivellement a des effets bénéfiques, car il permet de construire une recherche collective vivante, non planifiée, avec des échanges fructueux dépassant les relations hiérarchiques et ouverts au monde extérieur. Mais, en générant de nouvelles attentes, des possibilités décuplées de publications et de lecteurs, il aiguise aussi les crispations, les déceptions, les revirements. Être propulsé, que ce soit vers l'avant ou vers l'arrière, est déstabilisant.

Un autre effet contradictoire notable est celui de l'attente et de l'urgence : attente, car il n'est pas possible, ni souhaitable, de publier des résultats prématurés, qui risqueraient de 
s'avérer faux ; urgence, car un tel mouvement impose aussi des exigences de participation inhabituelle aux débats publics et laisse entrevoir des possibilités inédites de reconnaissance, et donc de légitimation. Pour reprendre l'image de la «spirale de l'engagement ethnographique », dont Daniel Céfaï (2010) montre que les différentes temporalités, du recueil des données à la controverse scientifique, s' " enroulent» les unes dans les autres, la recherche sur les Gilets jaunes donne l'exemple d'une spirale qui non seulement s'accélère mais peut s'inverser, en commençant par la controverse pour en arriver, des mois plus tard, au recueil des données.

\section{Conclusion}

Il est encore trop tôt pour dire à quels débats théoriques de fond donnera lieu cette ruée vers l'or jaune, avec tous ses courants et contre-courants. De la reconversion express au débarquement imprévu dans un univers qu'on connaît depuis des années, les trajectoires de recherche mettront du temps avant de se stabiliser autour d'interprétations durables. Mais on peut espérer que de ce foisonnement naîtront des pistes nouvelles pour la compréhension des luttes sociales. L'énigme d'un mouvement dont on ne trouve pas de signes précurseurs évidents pourra, par exemple, orienter la sociologie vers une démarche plus historique, attentive, notamment, aux transformations des mouvements sociaux et à leur inscription dans un temps long. Les enjeux économiques centraux posés par les revendications des Gilets jaunes, au sujet des taxes et des salaires, pourront quant à eux insuffler plus d'économie politique dans l'analyse des inégalités et, surtout, des social movement studies, qui se détournent depuis des années des conditions matérielles d'existence et de l'analyse de classe. Enfin, du point de vue du monde de la recherche, les pratiques d'interdisciplinarité à vif, de combinaison des méthodes et de collaborations inhabituelles entre générations, ont montré que la sociologie avait plus de sens quand elle s'emparait d'enjeux essentiels, en particulier de questions politiques qui la débordent, et qu'elle cherchait à transformer la science embarquée, déstabilisée par un objet qui la domine, en science engagée, qui décide consciemment de le penser, de l'intérieur. Cela suppose qu'on pense l'objectivité indépendamment de la neutralité qui, contrairement à ce qu'on entend parfois, peut être un sérieux obstacle à la compréhension du monde social.

\section{Bibliographie}

Bourdieu P. (1998 [1992]), Les Règles de l'art : genèse et structure du champ littéraire, nouvelle édition, revue et corrigée, Paris, Seuil.

Bourmeau S. (dir.) (2019), "Gilets jaunes » : hypothèses sur un mouvement, Paris, La

Découverte. 
Boyer P., Delemotte T., Gauthier G., Rollet V. \& Schmutz B. (2019), « Les déterminants de la mobilisation des "gilets jaunes" », documents de travail, Paris, CREST.

Burawoy M. (2009), The Extended Case Method. Four Countries, Four Decades, Four Great Transformations, and One Theoretical Tradition, Berkeley, CA, University of California Press.

Cefaï D. (dir.) (2010), L'Engagement ethnographique, Paris, Éditions de l'Ehess.

Challier R. (2018), « “Simples militants”. Sociologie comparée de l'engagement politique (FN, JC, UMP) en milieu populaire dans la France contemporaine », thèse de sociologie, Université Paris 8.

Challier R. (2019), « Rencontres aux ronds-points. La mobilisation des gilets jaunes dans un bourg rural de Lorraine ", La Vie des idées, 19 février

Collectif Plein le dos (2020), Plein le dos. 365 Gilets jaunes, sl, Les Éditions du bout de la ville.

Confavreux J. (dir.) (2019), Le fond de l'air est jaune : comprendre une révolte inédite, Paris, Seuil.

Coquard B. (2018), « Qui sont et que veulent les "gilets jaunes” ? Entretien avec Benoît Coquard », Propos recueillis par Ugo Palheta, Contretemps, 23 novembre, https://www.contretemps.eu/sociologie-gilets-jaunes/.

Coquard B. (2019), Ceux qui restent : faire sa vie dans les campagnes en déclin, Paris, La Découverte.

Desrosières A. (2014), La Politique des grands nombres. Histoire de la raison statistique, Paris, La Découverte.

Floris B. \& Gwiazdzinski L. (dir.) (2019), Sur la vague jaune : l'utopie d'un rond-point, Grenoble, Elya éditions.

Foucault M. (2017 [1963]), Naissance de la clinique, Paris, Puf.

Glaeser A. (2006), « An Ontology for the Ethnographic Analysis of Social Processes:

Extending the Extended Case Method », in Evens T. M. \& Handleman D. (dir.), The

Manchester School. Practice and Ethnographic Praxis in Anthropology, Oxford/New York,

Berghahn Books, p. 64-93.

Jeanpierre L. (2019), In girum. Les leçons politiques des ronds-points, Paris, La Découverte. L'Atelier des Ronds-Points (2019), « Conter et décrire les expériences de Gilets jaunes.

Journées d'études des 26 et 27 juin 2019 organisées par le IIAC, la MSH et le Laboratoire d'Innovation Sociale par la Recherche Action », Paris, MSH Paris Nord.

LISRA (sd), « Manifeste du Laboratoire d'Innovation Sociale par la Recherche-Action pour un tiers espace de la recherche et de l'action », Clichy, LISRA, http://recherche-

action.fr/labosocial/ docs/presentation-du-lisra/manifeste/.

Noiriel G. \& Truong N. (2019), Les Gilets jaunes à la lumière de l'histoire : dialogue avec Nicolas Truong, La Tour d'Aigues, Éditions de l'Aube.

Ravelli Q. (2015), La Stratégie de la bactérie. Une enquête au cour de l'industrie pharmaceutique, Paris, Seuil.

Ravelli Q. (2017), Les Briques rouges : logement, dettes et luttes sociales en Espagne, Amsterdam, Éditions Amsterdam.

Ravelli Q. (2019), « Debt Struggles: How Financial Markets Gave Birth to a Working-class Movement », Socio-Economic Review, mwz033, https://doi.org/10.1093/ser/mwz033. 
Ravelli Q. (2020), « Un chaos organisé. Les grappes de rondspoints comme structures politiques souples », Condition Humaine-Conditions Politiques, 1 (à paraître).

Ravelli Q., Bendali Z., Bonin L., Gaborit M., Grémion T., Ivanovic M., Liochon P. \& Toth N. (2020), « Le Gilet et le Marteau. L'Assemblée des assemblées organise l'aile gauche des rondspoints ", Mouvements, ${ }^{\circ} 101$, p. 13-24.

Spire A. (2018), Résistances à l'impôt, attachement à l'État : enquête sur les contribuables français, Paris, Seuil.

Spire A. (2019), « Reformuler la question sociale », in Confavreux J. (dir.) (2019), Le fond de l'air est jaune : comprendre une révolte inédite, Paris, Seuil, p. 91-98. 